\title{
COPD and muscle loss: is blood flow restriction a potential treatment?
}

\author{
Robert S. Thiebaud, Jeremy P. Loenneke, Takashi Abe
}

\begin{abstract}
Chronic obstructive pulmonary disease (COPD) is one of the leading causes of death in the United States and results in a significant reduction in lung function and exercise tolerance. In addition, there is a significant decline in muscle mass and strength in these individuals. Unfortunately, other comorbidities associated with this disease such as osteoporosis, osteoarthritis and obesity may prevent them from exercising at sufficiently high loads to promote muscle hypertrophy. Also, acute exacerbations may prevent them from performing exercise at all.

Objectives: This brief review will discuss the potential benefits of using blood flow restriction (BFR) when combined with walking, resistance training and electrical stimulation in COPD patients and possible safety concerns.

Design and Methods: Non-systematic review.

Results: BFR improves muscle size and function when combined with low-intensity walking or low-load resistance training. This treatment appears to be safe and has been used by many different populations including individuals with ischaemic heart disease. For COPD patients who are contraindicated to perform exercise, a potential treatment may be to combine neuromuscular electrical stimulation with BFR.

Conclusions: BFR appears to be a potential treatment for increasing strength and muscle mass for COPD patients when highintensity exercise may not be tolerated. In addition, BFR may provide benefits for COPD patients who are unable to exercise by combining it with neuromuscular electrical stimulation.
\end{abstract}

(Journal of Trainology 2014;3:1-5)

Key words: electrical stimulation $\boldsymbol{m}$ muscle hypertrophy $\boldsymbol{\bullet}$ lung disease $\boldsymbol{\bullet}$ pulmonary rehabilitation

\section{Introduction}

Chronic obstructive pulmonary disease (COPD) is currently the fourth leading cause of death in the United States and its prevalence around the world ranges from $4 \%$ in Hanover, Germany to $22 \%$ in Cape Town, South Africa. ${ }^{1}$ COPD is characterized by persistent decreased expiratory flow and dyspnea due to a remodeling of the small airways. ${ }^{2}$ The exact cause of COPD is complex and involves both genetic susceptibility and environmental factors but risk factors include cigarette smoke, maternal smoking, secondhand smoke exposure, childhood asthma, childhood respiratory infections, tuberculosis, outdoor air pollution, and occupational exposure to workplace irritants. ${ }^{3}$ Not only is this disease associated with impaired lung function, but also comorbidities such as cardiovascular disease, obesity, osteoporosis and osteoarthritis have been associated with it. ${ }^{4}$

One consequence of COPD is a significant decline in muscular strength and muscle mass. ${ }^{5}$ COPD patients have lower levels of upper leg strength and muscle cross-sectional area (CSA) compared to age-matched individuals. ${ }^{6,7}$ The decline in strength is particularly concerning as muscular weakness is correlated with decreased exercise performance and mortality in COPD patients. ${ }^{8,9}$ Furthermore, a decreased proportion of type I fibers and a decrease in CSA of both Type I, IIa, and IIab fibers are found in COPD patients compared to aged-matched healthy individuals. ${ }^{10}$ Several factors may explain the decline in muscle mass in this population such as increased oxidative stress, systemic inflammation, corticosteroid treatment, hypoxia and physical inactivity levels. ${ }^{5}$ However, COPD may take many years to develop before symptoms appear. Because of this, the age-related loss of muscle mass and function, also known as sarcopenia, may contribute to the loss of muscle mass/function in addition to the effects that COPD has on muscle mass. Therefore, the purpose of this short review will be to briefly discuss how traditional forms of exercise improve muscle size and function in COPD patients, and the potential use of combining BFR with exercise or electrical stimulation to enhance muscle hypertrophy in this population.

\section{Exercise or non-exercise treatment for patients with COPD}

\section{Exercise Treatments}

In order to overcome the loss of muscle mass and strength, several strategies have been used. One strategy has been continuous aerobic exercise. For example, continuous aerobic exercise at $50-70 \%$ of maximal power output increased peak

Received January 7, 2014; accepted January 28, 2014

From the Department of Health and Exercise Science, University of Oklahoma, Norman OK, USA (R.S.T, J.P.L), and Department of Kinesiology, Indiana University, Bloomington, IN USA (T.A.)

Communicated by Toshiaki Nakajima, MD, PhD (Guest Editor)

Correspondence to Robert S. Thiebaud, Department of Health and Exercise Science, The University of Oklahoma, 1401 Asp Ave., Norman, OK, 73019,

USA. E-mail: Robert.S.Thiebaud-1@ou.edu

Journal of Trainology 2014;3:1-5 @2012 The Active Aging Research Center http://trainology.org/ 
oxygen uptake by $11 \%$ and type I and IIa fiber CSA by $21-31 \%$ after 12 weeks of training. ${ }^{10}$ Another study found that after training for 10 weeks at $75 \%$ peak work rate, type I, type IIa and type IIb fibers CSA significantly increased by $10 \%$, $10 \%$, and $13 \%{ }^{11}$ Finally, Bernard et al. ${ }^{12}$ found a significant increase in quadriceps strength (8\%) but not thigh muscle CSA (3\%) after performing 12 weeks of aerobic training; however, combining aerobic training with strength training increased quadriceps strength significantly (20\%) and thigh CSA (8\%) more than aerobic only training. Not surprisingly, another study found that strength significantly increased more after strength training than endurance training in COPD patients..$^{13}$ Therefore, high-intensity aerobic training may improve strength and possibly muscle fiber size but to a lesser degree than resistance training. Unfortunately, some COPD patients cannot sustain continuous endurance exercise at high enough intensities to achieve these benefits due to several reasons. For example, earlier onset lactic acidosis, dynamic hyperinflation of the lungs, increased pulmonary vascular resistance and decreased right ventricular stroke volume can lead to earlier onset of muscle fatigue or exercise intolerance. ${ }^{14}$

Several studies have investigated the benefits of resistance training alone on muscular adaptations in COPD patients. Kongsgaard et al. ${ }^{15}$ had COPD patients perform high-intensity ( $80 \% 1 \mathrm{RM})$ lower body exercises for 12 weeks and found that both strength and mid-thigh quadriceps CSA increased while in the control group both actually declined. Another study examined the effects of only seven resistance training sessions $(70 \% 1 \mathrm{RM})$ and observed an increase in quadriceps strength and a decrease in myostatin mRNA expression. ${ }^{16}$ The ability to increase muscle mass and strength in COPD patients may be similar to healthy adults. To illustrate, Constantin et al. ${ }^{17}$ found that thigh lean mass and strength increased similarly to healthy subjects after performing 8 weeks of maximal isokinetic knee extensions. Furthermore, resistance training has been shown to not only increase strength but also exercise capacity. ${ }^{18}$ The current evidence suggests that high-intensity resistance training can be an important training mechanism for COPD patients to enhance muscle hypertrophy and strength when it is well tolerated by the individual. However, it is less clear whether resistance training also improves functional and maximal exercise capacity in COPD patients. ${ }^{19}$ Several concerns with resistance training may be that some COPD patients may not be able to perform such high-intensity resistance exercise due to an increased risk of muscle tears from chronic steroid treatment. ${ }^{14}$ Furthermore, those COPD patients who have osteoporosis may be contraindicated to perform high-intensity resistance exercise due to the high amount of stress placed on the joints.

\section{Non-exercise treatment}

A major advantage of a non-exercise treatment for COPD patients is that it does not produce any ventilatory stress during passive exercise. Neuromuscular electrical stimulation is a non-exercise treatment that has potential to increase strength and potentially muscle mass in COPD patients. When using electrical stimulation in rats, intracellular signaling pathways associated with muscle hypertrophy were activated, and significant increases in muscle mass and myofibrillar protein were found. ${ }^{20}$ In humans, Chaplin et al. ${ }^{21}$ found that electrically stimulating the quadriceps of hospitalized COPD patients for 30 minutes a day improved isometric quadriceps strength. In another study, hospitalized COPD patients had one leg randomly assigned to neuromuscular electrical stimulation and the other leg assigned as the control. After 14 days, the control leg had a decrease in strength by $3 \mathrm{~N}$ but the electrically stimulated leg increased strength by 19 N.22 One study also found that quadriceps strength, mid-thigh CSA and calf muscle CSA significantly increased after 6 weeks of neuromuscular electrical stimulation. ${ }^{23}$ These increases in muscle CSA, although significant, were only $6 \%$. Neuromuscular electrical stimulation could be a potential nonexercise treatment for COPD patients to improve strength and potentially muscle size without substantially increasing the stress on the respiratory system. However, the increases in muscle size and strength may not be very large. Therefore, because of a limited number of studies and the small changes, more evidence is needed before a definitive conclusion is drawn.

Due to acute exacerbations, limited physical ability or contraindications to performing high-intensity exercise, some patients with COPD may not be able to perform high-load exercise to induce muscle hypertrophy. In addition, some of the most common comorbidities associated with COPD are osteoarthritis and osteoporosis which may contraindicate some individuals from using high-intensity resistance training. One potential alternative to traditional forms of exercise may be blood flow restriction (BFR). BFR can be used without exercise to attenuate muscle atrophy and can be used with lowintensity or low-load exercise to significantly increase muscle size and strength. Therefore, it could be a major advantage for COPD patients who cannot handle the high-intensity or highload exercise.

\section{Blood flow restriction and COPD}

The novelty of BFR comes from the fact that significant muscle hypertrophy and strength gains are found when using it with low intensities or low loads. ${ }^{24}$ In addition, in the absence of exercise it can attenuate muscle loss. ${ }^{25}$ Both younger and older individuals have benefited from this technique as well as those recovering from injury ${ }^{25-27}$. Although few clinical populations have used this technique, it appears that it could be an alternative treatment for these individuals. ${ }^{28}$ The exact mechanism by which BFR enhances the increased muscle size and strength response to low-loads is not currently known. However, activation of the mechanistic target of rapamyocin (mTORC1) and mitogen activated protein kinase (MAPK) signaling pathways occurs with BFR and these appear to be key players involved with muscle hypertrophy ${ }^{29}$. In addition, decreased myostatin mRNA expression ${ }^{30}$, downregulation of proteolytic transcripts ${ }^{31}$, and proliferation of satellite cells ${ }^{32}$ occur with BFR. These events may be potentially activated through muscle cell swelling ${ }^{33}$ or metabolite buildup in the restricted exercising $\operatorname{limb}^{34}$. 


\section{Walking exercise with BFR}

Increases in muscle size and strength are found when BFR is combined with low-intensity exercises such as walking. For instance, Abe et al. ${ }^{35}$ found that muscle CSA and volume significantly increased by $4-7 \%$ and strength increased by $8-10 \%$ when walking $(50 \mathrm{~m} / \mathrm{min})$ was combined with BFR. In addition, Ozaki et al. ${ }^{36}$ found that older women who walked (45\% heart rate reserve) with BFR for 10 weeks increased muscle CSA and volume by $3-4 \%$ and strength by $6-22 \%$. Therefore, despite the low-intensities associated with walking, combining it with BFR improves muscle size and strength. This type of exercise may thus be advantageous for COPD patients who cannot perform intense aerobic exercise. However, the adaptations are considerably less for BFR combined with walking compared to BFR combined with resistance exercise.

\section{Resistance exercise with BFR}

With regards to combining BFR with resistance exercise, there is substantial evidence showing its beneficial effects on muscle size and strength. ${ }^{37}$ When physically active males performed low-load resistance exercise (20\%) with BFR, knee extension strength increased by $40 \%$ compared to $36 \%$ in the high-intensity group and $21 \%$ in the low-intensity only group. ${ }^{30}$ In addition, quadriceps CSA increased by $6 \%$ in both the low-intensity BFR group and high-intensity group. ${ }^{30}$ In older men and women, low-intensity resistance exercise combined with BFR has also been shown to significantly increase strength and muscle size. ${ }^{38-40}$ In addition, it has proved beneficial during rehabilitation from ACL surgery. After 16 weeks of combining BFR with traditional rehabilitation, knee extensor strength, knee flexor strength and knee extensor muscle CSA significantly recovered more compared to exercise without BFR. ${ }^{41}$ Interestingly, BFR exercise has also been noted to increase size and strength in muscle not directly under BFR (e.g. pectoralis major). ${ }^{42}$ It is thus a possibility that some respiratory muscles may be affected by BFR training but this is currently unknown. Therefore, when combining BFR exercise with low loads, muscle size and strength significantly increase and could be a useful strategy for COPD patients.

\section{Non-exercise with BFR}

Interestingly, improvements are found when BFR is applied in the absence of exercise. For instance, when low and high BFR pressures (50 and $200 \mathrm{mmHg}$ ) were used on unloaded limbs, a significantly smaller decline in muscular force was found than non-BFR limbs. ${ }^{43,44}$ Beneficial effects on attenuating muscle atrophy were also found in $\mathrm{ACL}$ reconstructive surgery patients..$^{25}$ In that study, an experimental group had BFR applied to the surgically repaired limb while the other group did not. The BFR pressure started at 180 $\mathrm{mmHg}$ and gradually increased during the next two weeks to levels varying from $200-260 \mathrm{mmHg}$. The stimulus was applied for five minutes and then removed for three minutes and repeated for five sets two times a day. After two weeks, a significant attenuation in the loss of muscle CSA was found for the BFR group versus the control group for men ( $9 \% \mathrm{vs}$.
$18 \%)$ and women (11\% vs. 23\%). ${ }^{25}$ Therefore, in COPD patients this may be a particularly useful treatment to attenuate loss of muscle and strength at times when exercise cannot be performed.

Taken as a whole, when BFR is combined with low-intensity walking or low-load resistance training, improvements in both muscular function and size happen and would be expected to also occur in COPD patients. What may be beneficial to treating the muscular declines found in COPD patients, especially those experiencing acute exacerbations, may be the application of BFR without exercise. In COPD patients, some studies have tried to electrically stimulate the muscle to produce muscular changes. A potentially new and exciting treatment for these individuals could be to combine BFR with electrical stimulation. BFR by itself in the absence of exercise attenuates the loss of muscle strength and size. One study noted that when combining BFR with neuromuscular electrical stimulation, a significant increase in metabolites was produced which lead to a greater release of serum immunoreactive growth hormone compared to the non-stimulated BFR limb. ${ }^{45}$ Thus, by electrically stimulating the muscle in combination with BFR, both muscle cell swelling and increased metabolites could occur potentially triggering a greater muscle hypertrophy response than neuromuscular electrical stimulation by itself. This is a future area of research that should be investigated.

\section{Methodology}

When using BFR it is important to recognize several methodological issues. Blood flow restriction involves placing a narrow cuff around the most proximal part of the exercising limb. The cuff is then inflated to a predetermined pressure with the intention to produce venous occlusion while restricting arterial inflow. Various methods have been used to produce this restrictive pressure but this produces problems when trying to compare studies ${ }^{46}$. Evidence shows that cuff pressures should be individualized based on the circumference of the limb and the size of the cuff being used. ${ }^{47}$ Several types of cuffs have been used such as elastic cuffs, nylon cuffs, and knee wraps. Larger cuff sizes produce significantly greater cardiovascular and perceptual responses compared to more narrow cuffs when a similar pressure is used ${ }^{48}$, and they produce arterial occlusion at lower pressures than narrow cuffs at rest ${ }^{47}$. However, when similar cuff sizes of different materials are used at the same target pressure, similar repetitions and exertion levels are found (unpublished data). In addition, similar arterial occlusion pressures are found at rest. ${ }^{49}$ These findings have been observed in young, healthy adults and future studies should investigate if a similar response would be found in an older and diseased population. Due to the individual differences in limb size and different physiological responses that can occur with different sized cuffs, it is very important that these things be considered when using BFR.

\section{Safety Concerns}

Currently, no studies have examined the safety of using BFR 
with COPD patients. Some potential concerns for COPD patients when exercising with BFR may be underlying cardiovascular disease and impaired respiratory capacity. When exercise is performed with BFR, a significant increase in oxygen consumption, heart rate, and blood pressure occur relative to the same exercise without BFR. ${ }^{50,51}$ It has been documented that different size cuffs can produce significant differences in cardiovascular responses so this should be considered when prescribing BFR exercise..$^{52}$ Despite this, in healthy adults very few side effects have been reported. ${ }^{53}$ Also, studies have shown that it may not affect markers of coagulation $^{54-56}$ but may acutely increase fibrinolytic potential ${ }^{54,56}$. More recently, patients with stable ischaemic heart disease who performed low-intensity exercise with BFR did not have significantly different changes in D-dimer and fibrinoghen/fibrin degradation products and high-sensitive $\mathrm{C}$-reactive protein compared to exercise at low-intensity exercise without BFR. ${ }^{28}$ Other concerns have been proposed such as increased oxidative stress potentially initiating greater muscle damage but a substantially greater increase in oxidative stress with BFR compared to high-intensity exercise has not been found. ${ }^{28,57}$ Although it is unlikely, more research is needed to verify if using BFR in severe COPD patients would damage skeletal muscle.

\section{Conclusion}

In conclusion, BFR may be a potential treatment for COPD patients for improving muscular size and function. COPD patients have significantly impaired respiratory capacity and some may be incapable of performing a substantial volume of exercise to improve muscle size and strength. However, combining BFR with electrical stimulation may be a potentially novel treatment to improve muscle size and function in these individuals.

\section{References}

1. Decramer M, Janssens W, Miravitlles M. Chronic obstructive pulmonary disease. Lancet 2012; 379: 1341-1351.

2. O'Donnell DE. Hyperinflation, dyspnea, and exercise intolerance in chronic obstructive pulmonary disease. Proc Am Thorac Soc 2006; 3: 180184.

3. Eisner MD, Anthonisen N, Coultas D et al. An official American Thoracic Society public policy statement: Novel risk factors and the global burden of chronic obstructive pulmonary disease. Am J Respir Crit Care Med 2010; 182: 693-718.

4. Reid WD, Yamabayashi C, Goodridge D et al. Exercise prescription for hospitalized people with chronic obstructive pulmonary disease and comorbidities: a synthesis of systematic reviews. Int J Chron Obstruct Pulmon Dis 2012; 7: 297-320.

5. Nasis I, Kortianou EA, Clini E et al. Effect of rehabilitative exercise training on peripheral muscle remodelling in patients with COPD: targeting beyond the lungs. Curr Drug Targets 2013; 14: 262-273.

6. Bernard S, LeBlanc P, Whittom F et al. Peripheral muscle weakness in patients with chronic obstructive pulmonary disease Am J Respir Crit Care Med 1998; 158: 629-634.

7. Seymour JM, Spruit MA, Hopkinson NS et al. The prevalence of quadriceps weakness in COPD and the relationship with disease severity. Eur Respir J 2010; 36: 81-88.

8. Swallow EB, Reyes D, Hopkinson NS et al. Quadriceps strength predicts mortality in patients with moderate to severe chronic obstructive pulmonary disease. Thorax 2007; 62: 115-120.

9. Gosselink R, Troosters T, Decramer M. Peripheral muscle weakness contributes to exercise limitation in COPD. Am J Respir Crit Care Med 1996; 153: 976-980.

10. Whittom F, Jobin J, Simard PM et al. Histochemical and morphological characteristics of the vastus lateralis muscle in patients with chronic obstructive pulmonary disease. Med Sci Sport Exer 1998; 30: 1467-1474.

11. Vogiatzis I, Terzis G, Nanas S et al. Skeletal muscle adaptations to interval training in patients with advanced COPD. Chest 2005; 128: 3838-3845.

12. Bernard S, Whittom F, Leblanc $P$ et al. Aerobic and strength training in patients with chronic obstructive pulmonary disease. Am J Respir Crit Care Med 1999; 159: 896-901.

13. Ortega F, Toral J, Cejudo P et al. Comparison of effects of strength and endurance training in patients with chronic obstructive pulmonary disease. Am J Respir Crit Care Med 2002; 166: 669-674.

14. Rochester CL. Exercise training in chronic obstructive pulmonary disease. $J$ Rehabil Res Dev 2003; 40: 59-80.

15. Kongsgaard M, Backer V, Jorgensen $\mathrm{K}$ et al. Heavy resistance training increases muscle size, strength and physical function in elderly male COPD-patients--a pilot study. Respir Med 2004; 98: 1000-1007.

16. Troosters T, Probst VS, Crul T et al. Resistance training prevents deterioration in quadriceps muscle function during acute exacerbations of chronic obstructive pulmonary disease. Am J Respir Crit Care Med 2010; 181: 1072-1077.

17. Constantin D, Menon MK, Houchen-Wolloff L et al. Skeletal muscle molecular responses to resistance training and dietary supplementation in COPD. Thorax 2013; 68: 625-633.

18. Vonbank K, Strasser B, Mondrzyk J et al. Strength training increases maximum working capacity in patients with COPD--randomized clinical trial comparing three training modalities. Respir Med 2012; 106: 557-563.

19. Grove TP. Should patients with chronic obstructive pulmonary disease be prescribed a resistance-training programme? Br J Sports Med 2010; 44: 396-397.

20. Tsutaki A, Ogasawara R, Kobayashi K et al. Effect of intermittent lowfrequency electrical stimulation on the rat gastrocnemius muscle. Biomed Res Int 2013; 2013: 480620.

21. Chaplin EJ, Houchen L, Greening NJ et al. Neuromuscular stimulation of quadriceps in patients hospitalised during an exacerbation of COPD: a comparison of low $(35 \mathrm{~Hz})$ and high $(50 \mathrm{~Hz})$ frequencies. Physiother Res Int 2013; 18: 148-156.

22. Giavedoni S, Deans A, McCaughey P et al. Neuromuscular electrical stimulation prevents muscle function deterioration in exacerbated COPD: a pilot study. Respir Med 2012; 106: 1429-1434.

23. Vivodtzev I, Debigare R, Gagnon P et al. Functional and muscular effects of neuromuscular electrical stimulation in patients with severe COPD: a randomized clinical trial. Chest 2012; 141: 716-725.

24. Loenneke JP, Wilson JM, Marin PJ et al. Low intensity blood flow restriction training: a meta-analysis. Eur J Appl Physiol 2012; 112: 18491859 .

25. Takarada Y, Takazawa H, Ishii N. Applications of vascular occlusion diminish disuse atrophy of knee extensor muscles. Med Sci Sports Exerc 2000; 32: 2035-2039.

26. Thiebaud RS, Loenneke JP, Fahs CA et al. The effects of elastic band resistance training combined with blood flow restriction on strength, total bone-free lean body mass and muscle thickness in postmenopausal women. Clin Physiol Funct Imaging 2013; 33: 344-352.

27. Yasuda T, Ogasawara R, Sakamaki M et al. Relationship between limb and trunk muscle hypertrophy following high-intensity resistance training and blood flow-restricted low-intensity resistance training. Clin Physiol Funct Imaging 2011; 31: 347-351.

28. Madarame H, Kurano M, Fukumura K et al. Haemostatic and inflammatory responses to blood flow-restricted exercise in patients with ischaemic heart disease: a pilot study. Clin Physiol Funct Imaging 2013; 33: 11-17.

29. Fry CS, Glynn EL, Drummond MJ et al. Blood flow restriction exercise 
stimulates mTORC1 signaling and muscle protein synthesis in older men. J Appl Physiol 2010; 108: 1199-1209.

30. Laurentino GC, Ugrinowitsch C, Roschel $\mathrm{H}$ et al. Strength training with blood flow restriction diminishes myostatin gene expression. Med Sci Sport Exer 2012; 44: 406-412.

31. Manini TM, Vincent KR, Leeuwenburgh CL et al. Myogenic and proteolytic mRNA expression following blood flow restricted exercise. Acta Physiol 2011; 201: 255-263.

32. Nielsen JL, Aagaard P, Bech RD et al. Proliferation of myogenic stem cells in human skeletal muscle in response to low-load resistance training with blood flow restriction. J Physiol 2012; 590: 4351-4361.

33. Loenneke JP, Fahs CA, Rossow LM et al. The anabolic benefits of venous blood flow restriction training may be induced by muscle cell swelling. Med Hypotheses 2012; 78: 151-154.

34. Yasuda T, Abe T, Brechue WF et al. Venous blood gas and metabolite response to low-intensity muscle contractions with external limb compression. Metab: Clin Exp 2010; 59: 1510-1519.

35. Abe T, Kearns CF, Sato Y. Muscle size and strength are increased following walk training with restricted venous blood flow from the leg muscle, Kaatsu-walk training. J Appl Physiol 2006; 100: 1460-1466.

36. Ozaki H, Sakamaki M, Yasuda $\mathrm{T}$ et al. Increases in thigh muscle volume and strength by walk training with leg blood flow reduction in older participants. J Gerontol A Biol Sci Med Sci 2011; 66: 257-263.

37. Loenneke JP, Wilson JM, Marin PJ et al. Low intensity blood flow restriction training: a meta-analysis. Eur J Appl Physiol 2012; 112: 18491859.

38. Karabulut M, Abe T, Sato $\mathrm{Y}$ et al. The effects of low-intensity resistance training with vascular restriction on leg muscle strength in older men. Eur J Appl Physiol 2010; 108: 147-155.

39. Takarada Y, Takazawa H, Sato Y et al. Effects of resistance exercise combined with moderate vascular occlusion on muscular function in humans. J Appl Physiol 2000; 88: 2097-2106.

40. Thiebaud RS, Loenneke JP, Fahs CA et al. The effects of elastic band resistance training combined with blood flow restriction on strength, total bone-free lean body mass and muscle thickness in postmenopausal women. Clin Physiol Funct Imaging 2013; 33: 344-352.

41. Ohta $\mathrm{H}$, Kurosawa $\mathrm{H}$, Ikeda $\mathrm{H}$ et al. Low-load resistance muscular training with moderate restriction of blood flow after anterior cruciate ligament reconstruction. Acta Orthop Scand 2003; 74: 62-68.

42. Abe T, Loenneke JP, Fahs CA et al. Exercise intensity and muscle hypertrophy in blood flow-restricted limbs and non-restricted muscles: a brief review. Clin Physiol Funct Imaging 2012; 32: 247-252
43. Kubota A, Sakuraba K, Sawaki K et al. Prevention of disuse muscular weakness by restriction of blood flow. Med Sci Sport Exer 2008; 40: 529534.

44. Kubota A, Sakuraba K, Koh S et al. Blood flow restriction by low compressive force prevents disuse muscular weakness. J Sci Med Sport 2011; 14: 95-99.

45. Inagaki $\mathrm{Y}$, Madarame $\mathrm{H}$, Neya $\mathrm{M}$ et al. Increase in serum growth hormone induced by electrical stimulation of muscle combined with blood flow restriction. Eur J Appl Physiol 2011; 111: 2715-2721.

46. Fahs CA, Loenneke JP, Rossow LM et al. Methodological considerations for blood flow restricted resistance exercise. $J$ Trainol 2012; 1: 14-22.

47. Loenneke JP, Fahs CA, Rossow LM et al. Effects of cuff width on arterial occlusion: implications for blood flow restricted exercise. Eur J Appl Physiol 2012; 112: 2903-2912.

48. Rossow LM, Fahs CA, Loenneke JP et al. Cardiovascular and perceptual responses to blood-flow-restricted resistance exercise with differing restrictive cuffs. Clin Physiol Funct Imaging 2012; 32: 331-337.

49. Loenneke JP, Thiebaud RS, Fahs CA et al. Effect of cuff type on arterial occlusion. Clin Physiol Funct Imaging 2013; 33: 325-327.

50. Kumagai K, Kurobe K, Zhong $\mathrm{H}$ et al. Cardiovascular drift during low intensity exercise with leg blood flow restriction. Acta Physiol Hung 2012; 99: 392-399.

51. Renzi CP, Tanaka H, Sugawara J. Effects of leg blood flow restriction during walking on cardiovascular function. Med Sci Sport Exer 2010; 42: 726-732.

52. Rossow LM, Fahs CA, Loenneke JP et al. Cardiovascular and perceptual responses to blood-flow-restricted resistance exercise with differing restrictive cuffs. Clin Physiol Funct Imaging 2012; 32: 331-337.

53. Nakajima T, Kurano M, Iida $\mathrm{H}$ et al. Use and safety of KAATSU training: results of a national survey. Int J KAATSU Training Res 2006; 2: 5-13.

54. Clark BC, Manini TM, Hoffman RL et al. Relative safety of 4 weeks of blood flow-restricted resistance exercise in young, healthy adults. Scand $J$ Med Sci Sports 2011; 21: 653-662.

55. Madarame H, Kurano M, Takano H et al. Effects of low-intensity resistance exercise with blood flow restriction on coagulation system in healthy subjects. Clin Physiol Funct Imaging 2010; 30: 210-213.

56. Nakajima T, Takano H, Kurano M et al. Effects of KAATSU training on haemostasis in healthy subjects. Int $J$ KAATSU Training Res 2007; 3: 11-20.

57. Goldfarb AH, Garten RS, Chee PD et al. Resistance exercise effects on blood glutathione status and plasma protein carbonyls: influence of partial vascular occlusion. Eur J Appl Physiol 2008; 104: 813-819. 